\title{
Taxonomy of curriculum change models: the case of Problem-Based Learning
}

\section{Modelli per introdurre innovazioni curriculari nella didattica universitaria: il caso del Problem-Based Learning (PBL)}

Antonella Lotti ${ }^{1}$

\begin{abstract}
The idea, proposed in this work, is that who are introducing educational innovation in University teaching should focus more on the renewal of a curriculum more than in a single course's changes.

University teaching innovation often focuses on the changes introduced within a single discipline through the adoption of some educational technologies or new teaching-learning-evaluation methods, keeping the focus only on the single discipline and not on the curriculum as a whole.

With reference to the work of Ronald Harden about the eight roles of the university teacher, we underline the role of Curriculum developer and implementer.

Six models are proposed to classify the introduction of innovative curricula.

The different ways by which Problem-Based Learning was introduced in university curricula are described as examples of the six models.
\end{abstract}

Keywords: curriculum, University educational innovation, Problem-Based Learning, curriculum developer.

\section{Introduzione}

La domanda di partenza di questo lavoro è se sia possibile ripensare il curricolo non solo come organizzatore di processi ma anche come elemento generativo di una visione olistica che motivi lo studente e dia senso ai percorsi disciplinari.

L'idea è di dedicare attenzione, nell'ambito dell'innovazione didattica universitaria, anche al rinnovamento del curricolo inteso come corso di laurea triennale, magistrale e di specializzazione.

\footnotetext{
${ }^{1}$ Ricercatore in Didattica e Pedagogia Speciale, Università di Genova.
} 
Alcuni studiosi si interrogano su quali siano i modelli curriculari più efficaci per introdurre il cambiamento nelle università e, quindi, si ritiene utile riflettere su una possibile classificazione o tassonomia dei cambiamenti curricolari (Snell, 2014).

Spesso l'innovazione didattica universitaria si concentra sui cambiamenti introdotti all'interno dei singoli corsi disciplinari tramite l'adozione di alcune tecnologie educative, o di strategie didattiche e valutative, mantenendo l'attenzione quindi solo sulla singola disciplina e non sul curricolo nel suo insieme.

Concordiamo con Harden e Lilley quando sostengono che tra gli otto ruoli del docente universitario, vi sia quello di Course developer and implementer, e in questa circostanza ci concentriamo sul secondo ruolo, quello di progettista del curricolo.

Proponiamo sei modelli che permettono di classificare l'introduzione dell'innovazione didattica nel curricolo, portando come studio di caso, l'adozione del metodo Problem-Based Learning.

\section{II ruolo del docente universitario come curriculum planner}

Alcuni studiosi, recentemente, hanno avviato ricerche descrittive qualitative e quantitative per illustrare quali siano i ruoli del docente universitario e hanno rilevato che il ruolo del docente universitario in realtà è molto sfaccettato e diversificato.

Nikendei, nel 2016, condusse una indagine su oltre duemila docenti universitari chiedendo loro di definirsi e identificò sei descrittori corrispondenti ai ruoli di facilitatore, fornitore di informazioni, pianificatore di curriculo, valutatore e creatore di strumenti di verifica, modello professionale e sviluppatore di risorse. Emersero altri ruoli quali: professionista esperto, entusiasta, formatore dei docenti universitari, formatore di studenti universitari di primo e secondo livello, ricercatore e amministratore.

Harden prese in considerazione lo studio di Nikendei e propose, recentemente, una versione che indica otto ruoli del docente universitario:

1. fornitore di informazioni e coach;

2. facilitatore e mentore;

3. progettista di curriculum (pianificatore e realizzatore);

4. valutatore e diagnostico;

5. role model come docente e professionista;

6. manager e leader;

7. studioso e ricercatore;

8. professionista. 
Fig. 1 - Gli otto ruoli del docente universitario di medicina (Harden and Lilley, 2018)

Gli 8 ruoli del docente universitario

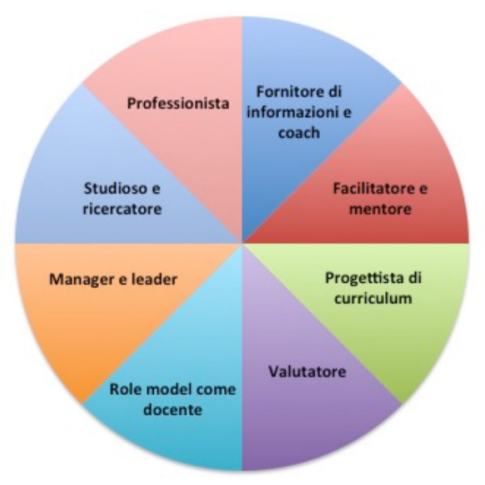

In questo contesto ci concentriamo sul ruolo di curriculum developer and implementer o progettista del curricolo.

Nel 1998 Diamond scrisse che l'attività che ha più impatto sulla didattica è quella intrapresa nella progettazione del curricolo e che la pianificazione del curricolo è di solito svolta da un gruppo di docenti in speciali commissioni dedicate (Diamond, 1998).

\section{II docente Curriculum Planner e le innovazioni didattiche universitarie}

Il docente universitario dovrebbe avere una competenza anche come progettista del curricolo in quanto egli è sollecitato da molteplici sfide e cambiamenti anche nel campo della didattica, primo tra tutti quello di formare professionisti ben preparati per un mondo che è dinamico e complesso e che richiede quindi di aver seguito un curriculo dinamico e flessibile.

Non tutti i docenti hanno l'opportunità, il tempo, la motivazione di introdurre un cambiamento nel proprio corso di laurea, ma ogni docente ha la possibilità di apportare un'innovazione all'interno del suo ambito: una lezione, un corso disciplinare, un corso di laurea, una istituzione accademica nel suo complesso. I docenti sono le persone-chiave per introdurre il cambiamento all'interno di un corso, i catalizzatori e i motivatori che possono portare avanti una vision nuova.

L'innovazione può riguardare elementi che hanno un minor impatto sul curricolo, come la ri-scrittura degli obiettivi formativi in termini di competenze e learning outcomes senza che questo cambiamento possa avere una ricaduta 
sull'apprendimento degli studenti e sul cambiamento della didattica, oppure può riguardare l'intero assetto del corso di laurea, come quando si introduce un progettazione per moduli interdisciplinari scardinando la didattica per discipline, o si introduce un approccio interprofessionale che prevede che gli studenti afferenti a corsi di laurea differenti apprendano insieme in modo attivo e interattivo in gruppi eterogenei per provenienza, o si adotta il metodo del problem based learning, e altro.

La scala dell'innovazione curricolare ha cinque gradini (vedi tabella n. 1).

Tab. 1 - I livelli della scala dellinnovazione curricolare (tratto da Harden and Lilley, 2018)

\begin{tabular}{|l|l|}
\hline Livello & Caratteristiche \\
\hline 1 & $\begin{array}{l}\text { "Nessun cambiamento", "Solo un'ombra", "tanto non servirà a niente": approccio dell'0- } \\
\text { strica }\end{array}$ \\
\hline 2 & $\begin{array}{l}\text { "Cambiamo le tende" - continuare come prima, ma con qualche parte modificata, come la } \\
\text { preparazione dei learning outcomes, ma nessun cambiamento nel programma di insegna- } \\
\text { mento/apprendimento }\end{array}$ \\
\hline 3 & $\begin{array}{l}\text { "Rinnovamento" - migliorare qualcosa che qualcuno sta già facendo, come rendere più } \\
\text { interattive e partecipate le lezioni e introdurre altre risorse per facilitare l'apprendimento }\end{array}$ \\
\hline 4 & $\begin{array}{l}\text { "Riforma" - aggiunta di nuovi elementi ai percorsi esistenti, come attività presso un centro } \\
\text { di simulazione di ateneo, attività di feedback sistematico o la flipped classroom }\end{array}$ \\
\hline 5 & $\begin{array}{l}\text { "Trasformazione" - realizzazione di qualcosa di completamente differente, come un curri- } \\
\text { colo che prevede apprendimento personalizzato e ritagliato sui bisogni individuali dei singoli } \\
\text { studenti }\end{array}$ \\
\hline
\end{tabular}

\section{Alcuni modelli per introdurre l'innovazione didattica nei curricula universitari}

L'introduzione dell'innovazione didattica intesa come ripensamento e trasformazione del curricolo, al fine di rendere le istituzioni formative realmente centrate sull'apprendimento, e non più sull'insegnamento, e orientate a favorire l'acquisizione di competenze, potrebbe essere categorizzata secondo sei modelli in relazione a quanto l'innovazione modifichi l'impianto del curricolo: se modifica solo l'insegnamento di qualche disciplina sparsa negli anni del corso di laurea lo chiamiamo "a morbillo"; se modifica un semestre o un anno lo chiamiamo "a moduli"; se modifica il curricolo nella sua interezza, ma solo per una percentuale di studenti lo chiamiamo "Parallel Track" o corso parallelo sperimentale; se modifica tutto il corso di laurea nella sua interezza lo chiamiamo "trasformazione di un corso tradizionale in innovativo"; se crea un corso di laurea di nuova istituzione in modo completamente innovativo lo chiamiamo "istituzione di un curricolo innovativo"; se introduce una innovazione interdiscipli- 
nare trasversale in tutti i semestri, utilizzando l'approccio dell'apprendimento basato sui problemi e organizzato per progetti, accanto a una didattica disciplinare, lo chiamiamo "Modello di Aalborg".

Per rendere più chiara l'illustrazione di questi modelli, prendiamo in considerazione il metodo Problem Based Learning e analizziamo la sua implementazione in sei diverse realtà. Il Problem Based Learning è un metodo didattico che si presta a essere introdotto come semplice metodo didattico accanto ad altre strategie formative, oppure può essere un organizzatore didattico, o dispositivo curriculare, che richiede una pianificazione di moduli interdisciplinari sia in porzioni di curricula (esempio: moduli o semestri); si presta anche alla sua adozione per interi corsi di laurea, sino ad arrivare a tutti i corsi di laurea di un ateneo (Lotti, 2018).

Fig. 2 - I sei modelli dell'innovazione curriculare

Tabella n. 4 : Modelli per le innovazioni curriculari universitarie

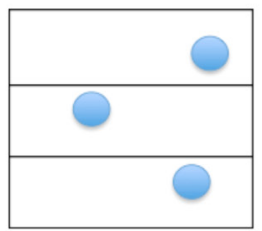

1. A morbillo

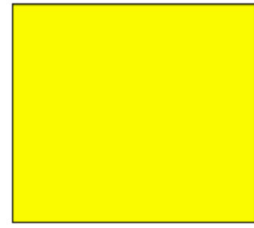

5. Trasformativo

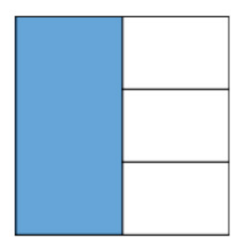

2. Parallel track

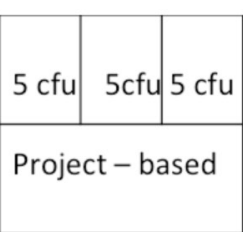

6. Modello di Aalborg

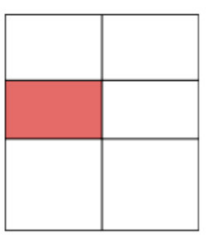

3. A modulo o a semestre

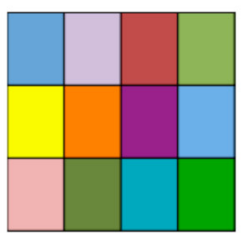

4. Innovativo a blocchi

\section{Il modello "a morbillo"}

L'introduzione di una innovazione educativa, all'interno di una singola disciplina, da parte di un docente è di solito abbastanza semplice perché il docente, in virtù della autonomia didattica universitaria, può pianificare e realizzare 
il suo insegnamento in piena libertà. Sono numerosi i docenti che sperimentano forme di insegnamento e apprendimento considerate innovative da se stessi e da altri. Ricerche svolte recentemente da otto università italiane, con la regia dell'Università di Padova, hanno rivelato che sono numerose le innovazioni introdotte nella didattica universitaria e che queste variano dall'utilizzo di nuovi software per far partecipare maggiormente gli studenti (esempio: Socrative, Kahoot, i clickers, i software specifici alle proprie discipline) all'utilizzo di visite guidate sul territorio presso aziende e stakeholders, alle testimonianze in aula da parte di esperti provenienti dal mondo del lavoro, da journal club a lavori in piccoli gruppi (Lotti, 2018). Prendendo in esame il Problem Based Learning nel modello a morbillo, ossia quando una innovazione didattica viene introdotta in una o più discipline all'interno dello stesso corso di laurea per qualche volta nell'arco dell'anno accademico, l'esempio che appare lampante è l'adozione del PBL nel corso di laurea di Infermieristica dell'Università di Genova dove i docenti di infermieristica propongono una o due sessioni di PBL agli studenti ogni semestre: gli studenti vengono divisi in piccoli gruppi di dieci persone e, condotti da un tutor, analizzano casi clinici, li discutono liberamente seguendo i "sette salti", individuano i sotto-problemi, formulano ipotesi esplicative o risolutive, formulano obiettivi di studio e vanno a studiare autonomamente. Dopo qualche giorno i gruppi si incontrano nuovamente con il tutor e ogni studente racconta ciò che ha trovato e studiato, integrando le sue conoscenze con quelle dei suo compagni, favorendo un apprendimento tipico del costruttivismo sociale.

Il metodo del PBL in questo modello è utilizzato come una strategia didattica innovativa all'interno di una disciplina che lo adotta solo un paio di volte in un semestre. Da una parte gli studenti hanno la possibilità di sperimentare un metodo attivo e interattivo, simile all'approccio che troveranno nel mondo del lavoro quando incontreranno problemi reali da affrontare in équipe professionali; dall'altra gli studenti si trovano a dover ricercare in modo autonomo e libero nei ritagli di tempo lasciati dalla frequenza alle lezioni delle altre discipline presenti nel curricolo. Essi sono sottoposti a più modelli didattici simultaneamente e questo provoca un certo disorientamento.

Il modello "a morbillo" evoca comunque l'immagine di una malattia infettiva e contagiosa e, in effetti, questa componente virale può rappresentare una modalità di disseminazione delle innovazioni nelle comunità di pratica e di ricerca dei docenti universitari. La narrazione di una propria esperienza in gruppi tra pari, o la sua condivisione in convegni scientifici o pubblicazioni di settore, certamente rappresentano una modalità di formazione tra pari e viene identificata come una potente via per l'introduzione del cambiamento (O'Sullivan and Irby, 2014). 


\section{Il modello del "Parallel Track"}

L'introduzione di un approccio centrato sull'apprendimento e non più sull'insegnamento rappresenta un cambio di paradigma molto difficile da attuare in istituzioni universitarie con una lunga tradizione alle spalle. Un metodo che favorisce l'introduzione del cambiamento all'interno di un corso di laurea, attraendo i docenti più aperti alle innovazioni didattiche, e permettendo di adottare lo stile di una sperimentazione scientifica è quello conosciuto con il nome di Binario Parallelo o Sperimentale o Alternativo (Experimental, Alternative o Parallel Track). In questo caso il corso di laurea approva che un gruppo limitato di studenti segua un percorso sperimentale parallelo dal primo all'ultimo anno. Esempi di Parallel Track li troviamo a livello internazionale e nazionale negli anni Ottanta e Novanta del secolo scorso nei corsi di laurea in Medicina e Chirurgia. Nel 1986 un gruppo formato da otto università, provenienti da cinque Paesi (USA, Cina, Tailandia, Filippine), si ritrovò ad Albuquerque in New Mexico per confrontare i risultati ottenuti adottando i Parallel Track come strategia per introdurre il cambiamento nella formazione dei futuri medici. In grandi università i Parallel track offrivano un corso alternativo a una coorte ridotta di studenti (6 a Michigan State, 20 a New Mexico, 40 a Harvard, 140 a Mexico) i quali studiavano in piccoli gruppi con il PBL e svolgendo tirocini precoci nelle strutture comunitarie sin dal primo anno. L'esempio più noto e prestigioso di parallel track fu quello dell'Università di Harvard che adottò il Parallel track per una coorte di studenti, chiamata Oliver Wendell Holmes Society. Dopo aver sperimentato nella Oliver Wendell Holmes Society sia il metodo del PBL che l'incontro precoce con i pazienti nella comunità, la Scuola di Medicina di Harvard adottò questo modello nella sua globalità e lo trasferì all'intero corso di laurea (Kantrowitz, 1987).

In Italia vi furono due Parallel Track: uno presso l'università di Bari a partire dall'AA 1988-89 (Palasciano, 1989) e uno presso l'Università di Roma La Sapienza a partire dall'AA 1993-94 (Fioravanti et al., 1997).

I corsi Paralleli o Sperimentali o Alternativi propongono curricula completamente differenti dal primo all'ultimo semestre, e coinvolgono tutti i docenti in pianificazioni interdisciplinari, con la richiesta di vedere il curricolo come un intero corso di laurea di cui tutti sono protagonisti e responsabili, al di là della propria disciplina.

\section{II modello "a moduli" (un semestre - un anno)}

Se da una parte il Parallel Track introduce una innovazione verticale, il "modello a moduli" introduce una innovazione in una porzione del curricolo in 
orizzontale e avviene quando un gruppo di docenti, che insegnano nello stesso semestre, decidono di intrecciare le proprie discipline e definiscono insieme gli obiettivi interdisciplinari da far raggiungere agli studenti, condividono e adottano le stesse strategie formative e valutative, creando un modulo didattico omogeneo e coerente per obiettivi, metodi formativi e valutazione (Domenici, 1998). Un esempio di Modulo innovativo è rintracciabile nel corso di laurea in Scienze della formazione continua dell'Università di Foggia quando nell'A.A. 2005-06 i docenti del primo semestre del secondo anno di corso decisero di adottare il PBL per cinque settimane al posto delle lezioni tradizionali.

I docenti di Antropologia culturale, Letteratura per l'infanzia, Didattica generale, Formazione a distanza, Pedagogia del lavoro fissarono insieme gli obiettivi irrinunciabili dei propri corsi, conseguentemente costruirono cinque situazioni - problematiche che avrebbero fatto acquisire le conoscenze irrinunciabili, pianificarono una serie di visite a strutture del territorio e alcune attività laboratoriali pertinenti, pianificarono un sistema di valutazione che tenesse conto degli apprendimenti disciplinari, delle competenze trasversali dimostrate nei lavori in piccolo gruppo durante il $\mathrm{PBL}$, la presenza e la partecipazione a tutte le opportunità formative proposte (Lotti, 2018).

\section{II modello "Istituzione di un curricolo innovativo ex-novo"}

Vi è un detto che recita che "è più facile trasferire un cimitero, che cambiare un curricolo" e, in effetti, la storia di alcune sperimentazioni sembra proprio confermare quanto sia più facile costruire un curricolo completamente innovativo laddove non esista un corso già insediato.

Le Università nuove, sia pubbliche che private, hanno maggior libertà nella pianificazione dei curricula e sono meno soggette a condizionamenti legati alla tradizione. Alcune innovazioni didattiche richiedono anche spazi diversi e gli edifici storici o quelli costruiti con grandi aule con sedie inchiodate a terra a volte impediscono la realizzazione di una didattica realmente partecipativa, attiva e interattiva. La costruzione degli edifici di una nuova università può rispondere a indicazioni architettoniche richiedenti ambienti per l'apprendimento cooperativo e in piccoli gruppi, centri di simulazione diffusi, stanze con arredi multifunzionali, laboratori didattici ispirati a nuovi principi educativi. Ad Aalborg in Danimarca, ad esempio, sono stati costruiti edifici che prevedono 1400 stanze per i lavori in piccoli gruppi. Quando fu fondata l'università di Maastricht in Olanda negli anni Settanta, il rettore e i docenti chiesero di costruire edifici nuovi con stanze per il PBL, skillslab o laboratori didattici ove apprendere in modo autonomo a contatto con manichini, simulatori, pazienti simulati, strumenti didattici di ultima generazione. Nella Facoltà di Giurisprudenza fu 
costruito un tribunale simulato, nella Facoltà di Psicologia furono costruiti lunghi corridoi ove si affacciavano stanze per ospitare gruppi di dieci studenti intorno a un tavolo per svolgere le sedute di PBL, nella Facoltà di Medicina fu costruito il primo Skills-Lab del mondo ove si trovavano decine di stanze attrezzate con manichini e simulatori, per apprendere le manovre semeiologiche e tecniche, e decine di stanze con specchi unidirezionali ed equipaggiate con sistemi di videoregistrazione per registrare i colloqui tra studenti e pazienti simulati al fine di sviluppare le abilità relazionali e comunicative.

In questi spazi fu più agevole introdurre curricula che erano organizzati per moduli interdisciplinari e che si avvalevano principalmente del metodo del PBL. A Maastricht in particolare le innovazioni curriculari riguardavano la pianificazione del curricolo per moduli di sei settimane, l'introduzione di metodi di valutazione innovativi come l'esame a stazioni e il progress test.

Lo studio delle Scuole universitarie innovative è stato oggetto di un libro che riporta i curricula di scuole istituite nei primi anni Settanta e dimostra come in quei contesti sia più semplice introdurre cambiamenti significativi (Richards et al., 1987).

Anche in Italia vi sono esempi di Università innovative che hanno adottato, ad esempio, il PBL e l'Inquiry Based Learning come metodo principale: il Campus Biomedico a Roma e Humanitas International School of Medicine a Milano.

\section{II modello "Trasformazione di un corso tradizionale in innovativo"}

Il modello più difficile da attuare è quello che prevede la trasformazione di un corso di laurea esistente in un corso completamente diverso, centrato sull'apprendimento.

Rari sono gli esempi di trasformazioni avvenute con successo all'interno di corsi di laurea tradizionali.

Tra questi ricordiamo due esperienze canadesi, entrambe nelle Facoltà di Medicina: quella di McMaster University nel 1971 e quella dell'Università di Sherbrooke in Quebec nel 1987.

In entrambi i casi i docenti passarono da un curricolo per discipline a un curricolo per moduli interdisciplinari integrati rispetto agli apparati anatomici (sistema locomotore, sistema digestivo, sistema cardiovascolare, sistema renale, ecc), al ciclo di vita (nascita, infanzia, adolescenza, età adulta, vecchiaia), ai grandi problemi complessi. Entrambe adottarono il Problem Based Learning in piccoli gruppi condotti da tutor. In questo caso è interessante analizzare le motivazioni che hanno spinto le due Università a cimentarsi in un cambiamento così radicale e trasformativo: nel primo caso a Mc Master University, il corpo 
docente si motivò a partire dalla consapevolezza che gli studenti erano frustrati e scontenti dopo le prime settimane di corso, si annoiavano a lezione, erano in una posizione passiva e si entusiasmavano solo qualche anno dopo, quando iniziavano ad andare in reparto a contatto con i pazienti. Il Comitato didattico decise di avviare una sperimentazione che prevedeva la pianificazione del curricolo in blocchi della durata di un mese e l'adozione di un metodo che presentava casi biomedici a partire dal primo anno, la suddivisione in piccoli gruppi, la guida di ogni gruppo da parte di un docente facilitatore (o tutor di PBL) e un'alta percentuale di tempo dedicato allo studio auto-diretto.

Nel secondo caso, quello di Sherbrooke, il timore di non essere all'altezza delle visite di accreditamento del Liason Committee e la consapevolezza che la didattica erogata non era coerente con le indicazioni dei documenti nazionali, che esortavano a sviluppare abilità di studio autonomo, ridurre il numero delle lezioni tradizionali per dar spazio ad attività di problem solving e studio indipendente, motivarono un gruppo di docenti della Facoltà di Medicina a realizzare nell'arco di due anni una trasformazione del curricolo che è entrata nella storia della pedagogia medica internazionale. La storia di Sherbrooke è entusiasmante e descrive come un corso di laurea tradizionale possa trasformarsi rapidamente introducendo il PBL come organizzatore curricolare e come metodo alternativo alla lezione tradizionale, scardinando la tradizionale divisione tra scienze precliniche e cliniche (Des Marchais, 2001).

\section{II modello di Aalborg}

Il modello pedagogico dell'Università di Aalborg in Danimarca, adottato dalla maggior parte dei corsi di laurea, da Ingegneria a Scienze umane, è ispirato al metodo Project-Oriented and Problem Based Learning. Il curricolo è organizzato in semestri della durata di 30 crediti, e in ogni semestre sono previsti tre insegnamenti da 5 crediti erogati con lezioni tradizionali e un blocco trasversale da 15 crediti in cui gli studenti, suddivisi in piccoli gruppi, con la guida di un docente tutor, affrontano e risolvono un problema reale tratto dal territorio, secondo l'approccio del Project-based learning e del Problem Based Learning.

A titolo di esempio si citano due casi di progetti che i piccoli gruppi di studenti devono risolvere.

Ad Ingegneria, ad esempio, si chiede di individuare una scuola sul territorio e di cercare di rendere l'edificio a risparmio energetico.

A Scienze Sociali si chiede ai gruppi di analizzare i dati sulla dispersione scolastica e sui drop-out quindicenni e di proporre un piano di rimedio. 
I gruppi lavorano in stanze dedicate tutto il tempo che desiderano: prima cercano di individuare il problema, poi formulano ipotesi, cercano informazioni per validare le loro ipotesi e alla fine propongono un progetto che risolva il problema. Questo progetto viene presentato a una commissione mista, composta da docenti universitari ed esperti esterni, che valuta sia il progetto nel suo insieme, sia la competenza del gruppo, sia le conoscenze individuali dei singoli membri del gruppo (Moesby, 2008).

\section{Fig. 3 - Il modello di Aalborg}

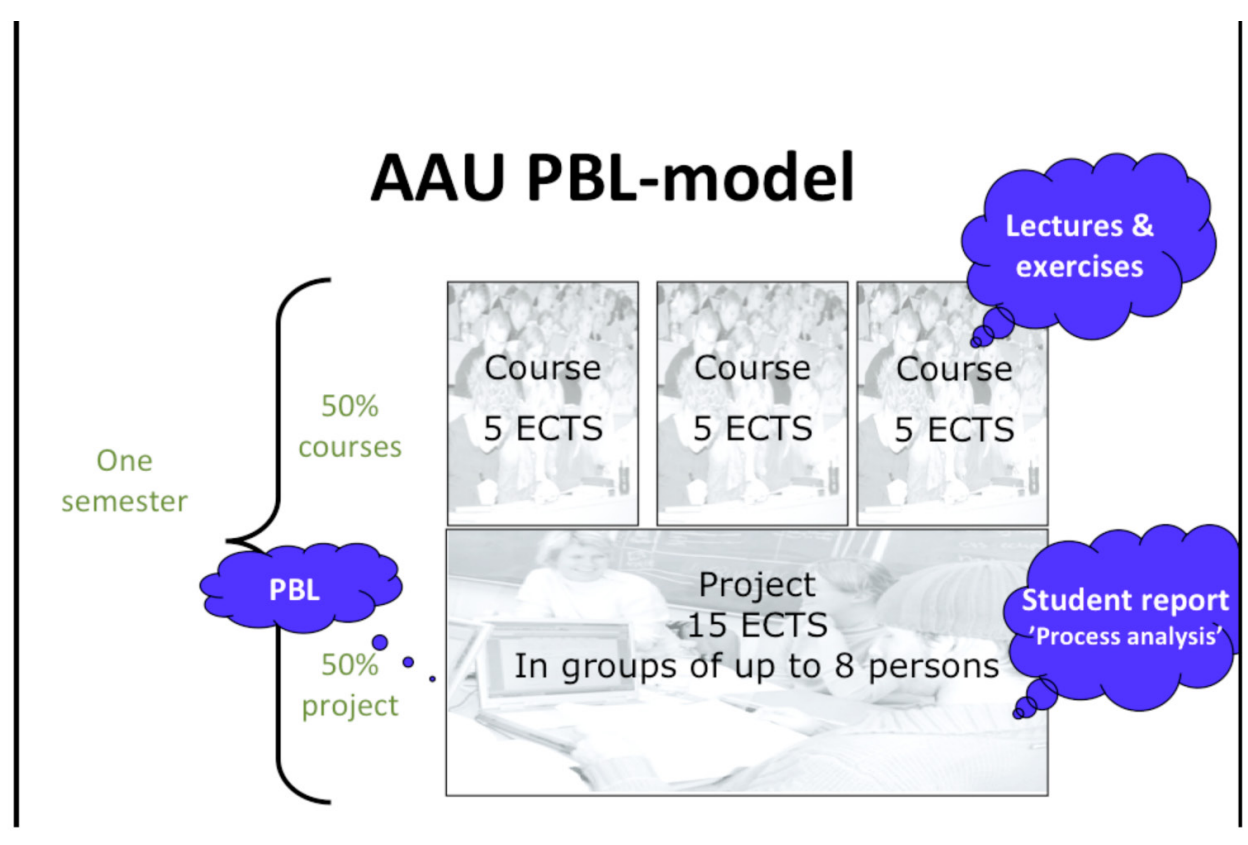

\section{Conclusioni}

Questo lavoro ha voluto focalizzare l'attenzione sull'innovazione curriculare come leva per introdurre il cambiamento all'interno della didattica universitaria soffermandosi sul ruolo del docente universitario come curriculum planner, piuttosto che come course planner. L'idea sostenuta è che il docente dovrebbe alzare lo sguardo e non soffermarsi solo sul suo insegnamento disciplinare (course planner), ma dovrebbe guardare al corso di laurea come un percorso unico di cui è responsabile insieme ai colleghi, come Team Teaching.

Dopo aver illustrato alcuni modelli possibili per introdurre l'innovazione a livello di curricolo, e aver portato esempi internazionali e nazionali di appli-

$$
164
$$


cazioni di queste innovazioni curriculari, dimostrando che l'innovazione curriculare è possibile anche a livello italiano, restano da approfondire alcune domande fondamentali: perché introdurre una innovazione curricolare? Quale valutazione delle innovazioni didattiche universitarie?

\section{Riferimenti bibliografici}

Des Marchais J.E. and Collaborators (2001). Learning to become a Physician at Sherbrooke. A Full Switch to a student-centered MD Program. Maastricht: Network Publications.

Diamond R.M. (1998). Designing and Assessing Courses and curricula: a practical guide, 2nd. San Francisco, USA: Jossey-Bass.

Domenici G. (1998). Manuale dell'orientamento e della didattica modulare. Bari: Laterza.

Fioravanti M., Gazzaniga P., Marcheggiano A., Osborn J., Renda T., Torsoli A. \& Ziparo V. (1997). The Rome Parallel Track (CPR). In A.J.J.A. Scherpbier et al. (eds.), Advances in Medical Education, Kluwer Academic Publishers, pp. 506-508

Harden R.M. \& Lilley P. (2018) The eight roles of the medical teacher. Elsevier Limited.

Kantrowitz M., Kaufman A., Mennin S., Fulop T., Guilbert J.-J. (1987). Innovative Tracks at established institutions for the education of health personnel. An experimental approach to change relevant to health needs. World Health Organization, Geneva (WHO Offset Publication n. 101).

Lotti A. (2018). Problem Based Learning: Apprendere per problemi a scuola: Guida al PBL per l'insegnante. Milano: FrancoAngeli.

McKimm J. \& Jones P.K. (2017). Twelve tips for applying change models to curriculum design, development and delivery. Medical Teacher, ISSN: 0142-159X (Print) 1466187X (Online)

Moesby E. (2010). What is an effective approach to introducing PBL/POPBL in an institution? A model form making the change to PBL/POPBL as an alternative approach for Higher Education Institutions. Department of Development and Planning, Aalborg University, Aalborg, Denmark.

O'Sullivan P.S. \& Irby D.M. (2014). Promoting scholarship in Faculty Development: relevant research paradigms and methodologies. In Steinert Y. (ed.), Faculty Development in the Health Professions. A Focus on research and practice. Dordrecht: Springer Science.

Palasciano G., Barnaba S., Lotti A. \& Albano M.G. (1989). Le curriculum parallèle experimental de la Faculté de Médecine de l'Université de Bari (Italie). MEDUCS, 2, 4, pp. 99-101.

Richards R. \& Fulop T. (1987). Innovative schools for health personnel. Report of ten schools belonging to the Network of Community-Oriented Educational Institution for Health Sciences. Geneva: World Health Organization (WHO Offset Publication n. 102).

Snell L. (2014). Faculty Development for curriculum change: towards Competencybased education teaching and assessing. In Steinert Y. (ed.), Faculty Development in the Health Professions. A Focus on research and practice. Dordrecht: Springer Science. 